\title{
Frequency and Causes of Pediatric Ovarian Torsion A Systematic Review
}

\author{
Saira islam $^{1} \quad$ Sayeeda Khadija-tul-Sughra Marrium ${ }^{2} \quad$ Aleena Batool $^{2} \quad$ Hasham hafeez hanjra $^{3}$ \\ Adeel kiyani ${ }^{3}$ \\ University Institute of Radiology Science and Medical Imaging Technology, Faculty of Allied Health Science \\ University of Lahore
}

\begin{abstract}
Objective: The aim of my study was to determine the frequency and causes of pediatric ovarian torsion.

Background: The condition in which an ovary twist along the ligament which hold it in place is known as Ovarian Torsion. The blood flow to ovary and fallopian tube could be cut off because of this twisting. ovarian torsion cab be a cause of unbearable pain due to lack of sufficient blood that should be received by ovary. On behalf of this systematic review, I wanted to dictate the frequency and causes of pediatric ovarian torsion. On the basis of past studies, I reviewed ovarian torsion can be happen at any paediatric age (infant to eighteen years). Around fiftytwo $\%$ of the cases in children happen between nine years to fourteen years of age, with a median of age 11.

Method: An electric database search was performed (google scholar, PubMed and science direct) with time limit until January 2020. All studies, fully available in English, assessing the frequency and causes of pediatric ovarian torsion.

Study design: Systematic review-based design is used for this study.

Results: 16 articles were reviewed, I found that frequency of pediatric ovarian torsion were rare and happened at any pediatric age. Ovarian torsion was caused by benign lesion, malignancy or by normal ovary.

Purpose: The aim of my study was to determine the frequency and causes of pediatric ovarian torsion.

Keywords: Ovarian torsion, oophorectomy, neoplasm, malignancy.

DOI: $10.7176 / \mathrm{JHMN} / 90-15$

Publication date:June $30^{\text {th }} 2021$

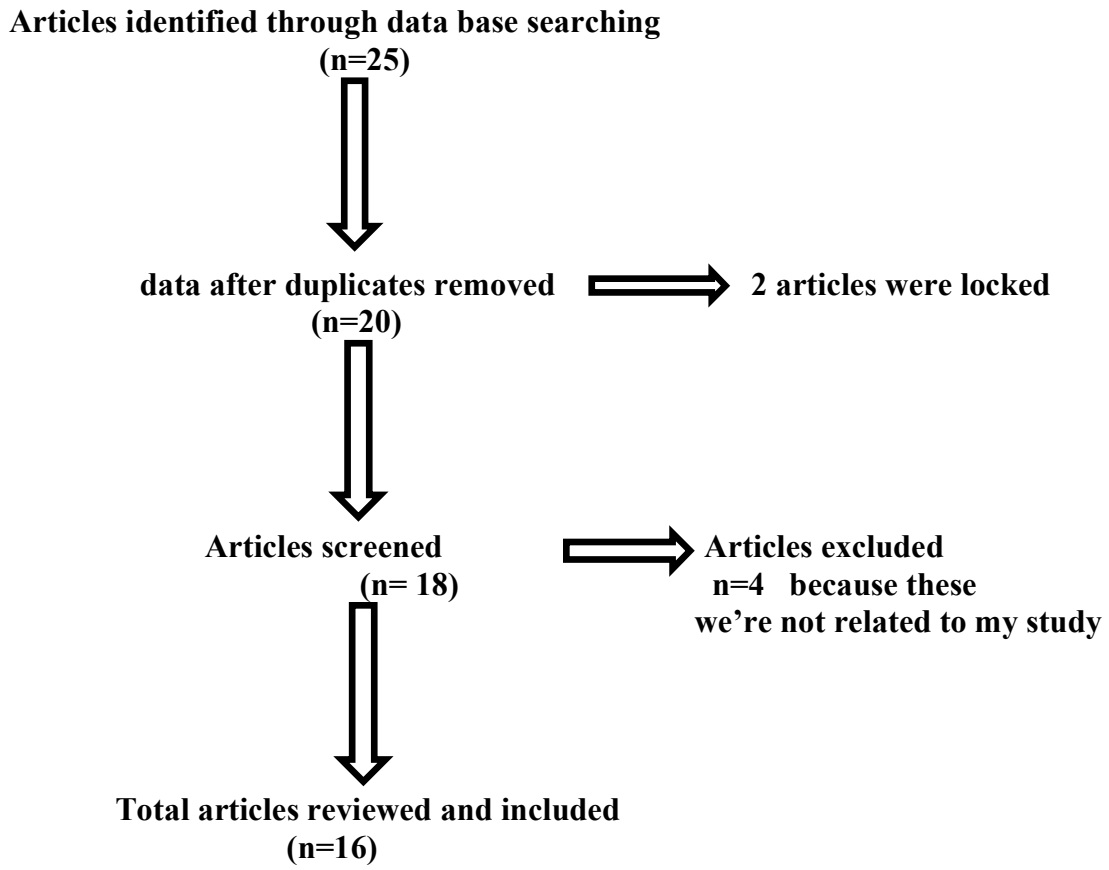

\section{Introduction}

Pediatric ovarian torsion accounts for round about $15 \%$ of all cases of ovarian torsion. ${ }^{1}$ Ovarian torsion can happen at any paediatric age (infants to eighteen years). Up to fifty-two \% of torsion cases in children happen between 9 years and 14 years old, with a middle age of 11 years. ${ }^{2}$ Ovarian torsion can be an abdominal catastrophe for females, especially if ovarian retrieve is not possible. Pathophysiological consequence include ovarian loss, intraabdominal infection, sepsis, and even death. The psychological effects can also be profound. ${ }^{3}$ Pediatric ovarian torsion is a quite rare occurrence with prospects of significant morbidity and possible mortality if not treated 
emergently. Pediatric ovarian torsion can be difficult to diagnose. ${ }^{4}$ Ovarian torsion is one of the most ordinary gynecological surgical emergencies. ${ }^{5}$ Torsion can be incomplete or complete, with some degrees of impaired circulation. Subsequently the sonographic appearance is affected by the onset and degree of torsion. ${ }^{6}$ The most common ultrasonographic finding is the presence of an enlarged ovary, with or without a mass, and fluid in culde-sac, which is generally a late presentation. ${ }^{7}$ Color Doppler for identification of torsion remains disputed because of the double blood supply to the ovary. ${ }^{8}$ Sometimes an ovarian twisted pedicle can be identified ("whirlpool sign"). ${ }^{9}$ It is more often seen in females of conceptive age, with nearly seventy-five \% developing in females between the ages of twenty and forty (mean 32-33.5) years, although it can happen at any age. ${ }^{10}$

\section{Discussion:}

In 2008, Chang YJ done a study. He reviewed the medical records of all cases of children whose adnexal torsion diagnosis was proven by surgery from 1992 to 2005 . Neonatal cases were excluded. A total of 49 cases were included in this study, with a mean age of 12.5 years. Sixteen $(32.6 \%)$ cases were premenarchal girls, who were more likely to have adnexal torsion missed at first clinical diagnosis when compared with post-menarchal girls $(P=0.032)$. Presenting symptoms included severe abdominal pain $(32 \%)$ and a history of recurrent pain of more than 2 weeks (14.2\%). Abdominal gray-scale ultrasound (US) in 43 patients revealed 41 cases that were suggestive of ovarian pathology and identified 3 torsions. Seventeen patients had both US and abdominal computed tomography, but no definitive adnexal torsion was diagnosed with the combined studies. When multiple radiographic studies were used, there was a significantly longer time from studies to operation as compared with US alone. ${ }^{11}$

A retrospective review of females aged 2-21 y who underwent an operation for an ovarian cyst or neoplasm between 2010 and 2016 at children's hospitals was performed. Of 814 girls with an ovarian neoplasm, 180 (22\%) had torsion. In risk-adjusted analyses, patients with a younger age, mass size $>5 \mathrm{~cm}$, abdominal pain, and vomiting had an increased likelihood of torsion $(P<0.01$ for all). Patients with a mass $>5 \mathrm{~cm}$ had two times the odds of torsion (odds ratio: 2.1; confidence interval: 1.2,3.6). Imaging was not reliable at identifying torsion (sensitivity $34 \%$, positive predictive value $49 \%$ ) or excluding torsion (specificity $72 \%$, negative predictive value $87 \%$ ). The rates of malignancy were lower in those with an ovarian mass and torsion than those without torsion $(10 \%$ versus $17 \%, P=0.01)$. Among the 180 girls with torsion and a mass, $48 \%$ underwent oophorectomy of which $14 \%(n=12)$ had a malignancy. ${ }^{12}$

A retrospective cohort study design was used. The medical charts of all premenarchal girls with surgically verified ovarian torsion treated in a university-affiliated tertiary medical center from 1997 to 2012 were reviewed for clinical, treatment, and outcome data. Thirty-two premenarchal girls were identified. Their median age was 9 years. There were 7 recurrences during the study period (17.9\%), for a total of 39 cases. The main presenting symptoms were abdominal pain $(92.3 \%)$ and nausea and vomiting $(84.6 \%)$. Physical examination revealed abdominal tenderness in 25 cases (64.1\%). Abdominal ultrasound, performed in 31 patients (38 cases), yielded pathologic findings in $28(73.7 \%)$, mainly an enlarged ovary (11 cases, 28.9\%). Doppler flow studies were abnormal in 15 cases. In 26 cases $(68.4 \%)$, the tentative preoperative working diagnosis was ovarian torsion. ${ }^{13}$

In 2009, Oltmans SC done a study. It was a 15-year review. Of 328 operative ovarian cases, 97 (29.6\%) demonstrated torsion. Mean patient age was 9.2 years (2 days to 17 years, \pm 0.54 SEM), with $52 \%$ occurring between 9 and 14 years. Of the patients, 97\% presented in pain. Presence of a pelvic mass $5 \mathrm{~cm}$ or larger on imaging had $83 \%$ sensitivity for torsion: an ultrasound reading was only $51 \%$ sensitive. Elevated white blood test count was the only preoperative characteristic associated with prompt operative intervention. ${ }^{14}$

This was a cohort analysis of the Healthcare Cost and Utilization Project Kids' Inpatient Database (KID) 2000, 2003, and 2006. Among females aged 1 to 20 years, there were 1232 cases of ovarian torsion, an estimated incidence of 4.9 per 100000 . A total of $713(58 \%)$ were treated with oophorectomy. The rate of ovarian torsionassociated oophorectomy remained unchanged from 2000 to 2006 . Fewer than $0.5 \%$ of ovarian torsion hospitalizations were associated with malignant neoplasm. ${ }^{15}$

In 2010, Huang R done a study and objective of this study was Pediatric ovarian malignancy presenting as ovarian torsion: incidence and relevance A total of 114 patients (mean \pm SEM age, 10 years, 2 days to 19 years \pm $0.53)$ with operatively proven ovarian torsion were identified. Four malignancies $(3.5 \%)$ and 26 benign neoplasm $(23 \%)$ were present in this age group. ${ }^{16}$

\section{Conclusion}

The purpose of this review was to view the frequency and causes of pediatric ovarian torsion. It is clear from the systematic review that ovarian torsion is rare and happens in any pediatric age and associates with no any abnormality in ovaries or benign lesions or ovarian malignancy. 
Images:

Figure 1:

Representative ultrasonographic images of a twisted ovary (a) and normal left ovary (b) in an 8-year-old girl presenting with pain in right lower abdomen. (a)The twisted ovary is greater in size and heterogeneous and measures $7.9 \times 3.8 \times 6.3 \mathrm{~cm}$ corresponding to a volume of $361.6 \mathrm{~cm}^{3}$. (b) The left normal ovary measures $2.8 \times 1.4 \times 1.9 \mathrm{~cm}$ corresponding to a volume of $14.2 \mathrm{~cm}^{3} .1$

Figure 2:

Whirlpool sign in ovarian torsion. a, b Color flow on US images identifies the twisted pedicle (arrows) in a twelveyear-old girl with a huge, mature cystic teratoma $(\mathrm{T})$ emerging from the left adnexa, representing the lead point for left adnexal torsion. ${ }^{2}$

\section{Reference:}

1. Servaes S, Zurakowski D, Laufer MR et al (2007) Sonographic findings of ovarian torsion in children. Pediatr Radiol 37:446-451

2. Oltmann SC, Fischer A, Barber R et al (2009) Cannot exclude torsion - a 15-year review. J Pediatr Surg 44:1212-1216, discussion 1217

3. Breech LL, Hillard PJ. Adnexal torsion in pediatric and adolescent girls. Current Opinion in Obstetrics and Gynecology. 2005 Oct 1;17(5):483-9 
4. Corre A, Dandekar S, Lau C, Ranasinghe L. A Case Report of Pediatric Ovarian Torsion: The Importance of Diagnostic Laparoscopy. Clinical Practice and Cases in Emergency Medicine. 2021 Feb;5(1):109.

5. Hibbard LT. Adnexal torsion. American journal of obstetrics and gynecology. 1985 Jun 15;152(4):456-61.

6. Rosado Jr WM, Trambert MA, Gosink BB, Pretorius DH. Adnexal torsion: diagnosis by using Doppler sonography. AJR. American journal of roentgenology. 1992 Dec;159(6):1251-3

7. Kokoska ER, Keller MS, Weber TR. Acute ovarian torsion in children. The American journal of surgery. 2000 Dec 1;180(6):462-5.

8. Spinelli C, Buti I, Pucci V, Liserre J, Alberti E, Nencini L, Alessandra M, Lo Piccolo R, Messineo A. Adnexal torsion in children and adolescents: new trends to conservative surgical approach-our experience and review of literature. Gynecological Endocrinology. 2013 Jan 1;29(1):54-8.

9. Lee EJ, Kwon HC, Joo HJ, Suh JH, Fleischer AC. Diagnosis of ovarian torsion with color Doppler sonography: depiction of twisted vascular pedicle. Journal of Ultrasound in Medicine. 1998 Feb;17(2):83-9.

10. Houry D, Abbott JT. Ovarian torsion: a fifteen-year review. Annals of emergency medicine. 2001 Aug $1 ; 38(2): 156-9$.

11. Chang YJ, Yan DC, Kong MS, Wu CT, Chao HC, Luo CC, Hsia SH. Adnexal torsion in children. Pediatric emergency care. 2008 Aug 1;24(8):534-7.

12. Lawrence AE, Fallat ME, Hewitt G, Hertweck P, Onwuka A, Afrazi A, Aldrink JH, Bence C, Burns RC, Corkum KS, Dillon PA. Factors Associated with Torsion in Pediatric Patients with Ovarian Masses. Journal of Surgical Research. 2021 Jul 1; 263:110-5.

13. Ashwal E, Krissi H, Hiersch L, Less S, Eitan R, Peled Y. Presentation, diagnosis, and treatment of ovarian torsion in premenarchal girls. Journal of pediatric and adolescent gynecology. 2015 Dec 1;28(6):526-9.

14. Oltmann SC, Fischer A, Barber R, Huang R, Hicks B, Garcia N. Cannot exclude torsion-a 15-year review. Journal of pediatric surgery. 2009 Jun 1;44(6):1212-7.

15. Guthrie BD, Adler MD, Powell EC. Incidence and trends of pediatric ovarian torsion hospitalizations in the United States, 2000-2006. Pediatrics. 2010 Mar 1;125(3):532-8.

16. Oltmann SC, Fischer A, Barber R, Huang R, Hicks B, Garcia N. Pediatric ovarian malignancy presenting as ovarian torsion: incidence and relevance. Journal of pediatric surgery. 2010 Jan 1;45(1):135-9.

\section{Image references:}

(1) Servaes S, Zurakowski D, Laufer MR, Feins N, Chow JS. Sonographic findings of ovarian torsion in children. Pediatric radiology. 2007 May;37(5):446-51.

(2) Ngo AV, Otjen JP, Parisi MT, Ferguson MR, Otto RK, Stanescu AL. Pediatric ovarian torsion: a pictorial review. Pediatric radiology. 2015 Nov;45(12):1845-55. 